\title{
Língua brasileira de sinais e implante coclear: relato de um caso
}

\author{
Michelle Nave Valadao* \\ Jilma Andrade Nomura** \\ Debora Hungria Mazer*** \\ Myriam de Lima Isaac ${ }^{\star \star \star *}$
}

\section{Resumo}

A perda auditiva é uma das privações sensoriais mais comum nos homens. A principal consequência da perda auditiva reside em sua repercussão no desenvolvimento da linguagem oral e, consequentemente, interferindo em todo o processo de aprendizagem da leitura e escrita. O implante coclear é um dispositivo eletrônico, parcialmente implantado, que visa a proporcionar aos seus usuários sensação auditiva próxima ao fisiológico. É uma boa opção aos portadores de surdez sensorioneural profunda, entretanto, não assegura o desenvolvimento das habilidades auditivas e de linguagem oral. Assim, por não desenvolverem a linguagem oral, os indivíduos com implante coclear não podem ser privados da possibilidade da aquisição e do desenvolvimento da linguagem, pois podem fazer isso utilizando outra forma de comunicação - a língua de sinais. O objetivo deste relato é descrever a experiência do atendimento multidisciplinar de uma criança usuária de implante coclear. B.V.S.R. oito anos, gênero masculino, portador de perda auditiva sensorioneural profunda, bilateral desde o período neonatal. Realizou cirurgia de implante coclear aos três anos de idade, sendo acompanhado segundo abordagem aurioral. Devido ao baixo desempenho escolar apresentado, foi discutido com a equipe multidisciplinar e decidido modificar a abordagem, optando-se por iniciar aprendizagem de língua brasileira de sinais. Após um período de dois anos, segundo a nova abordagem, a criança apresentou considerável evolução em todos os aspectos do desenvolvimento. A mudança de abordagem influenciou positivamente na evolução da criança, pois a mesma alcançou adequado desempenho em diversas habilidades linguísticas.

Palavras-chave: Surdez. Implante coclear. Língua brasileira de sinais.

\footnotetext{
* Fonoaudióloga do Hospital das Clinicas de Ribeirão Preto da Universidade de São Paulo (HCRP. USP), Doutora em Neurociências e Ciências do Comportamento pela Departamento de Neurociências e Ciências do Comportamento da Faculdade de Medicina de Ribeirão Preto da Universidade de São Paulo (FMRP-USP). Ribeirão Preto, São Paulo, Brasil.

** Jilma de Andrade Nomura, Pedagoga do Hospital das Clínicas de Ribeirão Preto da Universidade de São Paulo (HCRP-USP), especialista em audiocomunicação, intérprete em Língua Brasileira de Sinais (LIBRAS). Ribeirão Preto, São Paulo, Brasil.

*** Debora Hungria Mazer, Fonoaudióloga Clínica. Ribeirão Preto, São Paulo, Brasil.

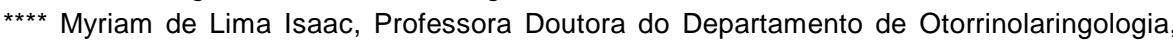
Oftalmogolgia e Cirugia de Cabeça e Pescoço da Faculdade de Medicina de Ribeirão Preto da Universidade de São Paulo (FMRP-USP). Ribeirão Preto, São Paulo, Brasil.
} 
Michelle N. Valadao - Jilma A. Nomura - Debora H. Mazer - Myriam de L. Isaac

\title{
Brazilian sign language and cochlear implant: case report
}

\begin{abstract}
The auditory loss is one of the more common sensorial privations in the men. The main consequence of the auditory loss inhabits consequent in its repercussion on the development of the verbal language and intervening with all the process of learning of the reading and writing. The cochlear implant is an electronic device, partially implanted, that it aims at to provide to its users next auditory sensation the physiological one. Deep interim is a good option to the carriers of neurossensorial deafness, it does not assure the development of the auditory abilities and verbal language. Thus, for not developing the verbal language, the individuals with cochlear implant cannot be private of the possibility of the acquisition and the development of the language; therefore they can make this using one another form of communication - the language of signals. The objective of this story is to describe the experience of the attendance to multidiscipline of a using child of cochlear implant. B.V.S.R eight years, masculine, carrying sort of bilateral deep neurossensorial loss auditory since the neonatal period. It carried through surgery of cochlear implant to the three years of age being folloied according to aurioral boarding. Had to presented overhead, case with the team was argued to multidiscipline and determined to modify the boarding, opting itself to initiating learning of Brazilian Sign Language. After a period of two years according to new boarding, the child presented considerable evolution in all the aspects of the development. The boarding change influenced positively in the evolution of the child, therefore the same one reached adequate performance in diverse linguistic abilities.
\end{abstract}

Keywords: Deafness. Cochlear implant. Brazilian Sign Language.

\section{Introdução}

A perda auditiva é o déficit sensorial mais comum e resulta na restrição das habilidades de se comunicar pela linguagem falada. Uma em cada mil crianças nasce surda ou se torna portadora de surdez profunda ou severa antes que a linguagem seja adquirida (período pré-lingual). Outras duas ou quatro crianças em cada 1000 se tornarão surdas ou portadoras de perda auditiva antes da vida adulta. Nos países desenvolvidos, mais de $50 \%$ da surdez na infância é atribuída a causas genéticas (GODINHO; KEOGH; EAVEY, 2003).

Por não ser de notificação compulsória aos órgãos públicos, a estimativa de sua incidência é difícil em nosso país. Em 1995 a World Health Assembly (WHA) estimou em 120 milhões o número de deficientes auditivos e em $2.2 \%$ a prevalência da surdez na população mundial (BENTO et al. 2004).

As alterações auditivas podem ser causadas por diversos fatores etiológicos, congênitos ou adquiridos, além de apresentar graus e tipos variáveis. Dentre as causas da deficiência auditiva adquirida, encontra-se o uso de 
algumas substâncias ototóxicas, que provocam perturbações transitórias ou definitivas das funções auditiva e vestibular (JACOB et al. 2006).

Determinados medicamentos têm a capacidade de provocar lesões no ouvido interno, acarretando surdez sensorioneural. A hipoacusia geralmente aparece no decurso da administração do medicamento, mas pode surgir tardiamente, o que é menos frequente, e evoluir mesmo depois de interrompido tratamento (KÓS; KÓS 2003).

A prótese auditiva convencional é eficaz no tratamento de grande parte das deficiências auditivas. Porém, por ser um amplificador sonoro, a prótese auditiva necessita de uma reserva coclear suficiente para que possa haver uma boa percepção do som e da fala. Alguns indivíduos, porém, apresentam uma disfunção auditiva tão importante que mesmo uma prótese auditiva potente não consegue ajudá-los a obter discriminação de palavras e sentenças. Indivíduos que não alcançam uma discriminação maior que $40 \%$ em testes de reconhecimento de sentenças em apresentação aberta, com a melhor amplificação auditiva possível, são candidatos a uma segunda opção de dispositivo eletrônico de amplificação sonora: o implante coclear (BENTO 2004; MORET; BEVILACQUA; COSTA-FILHO, 2007).

Os implantes cocleares são dispositivos eletrônicos, biomédicos, de alta tecnologia, desenvolvidos para realizar a função das células ciliadas da cóclea que estão danificadas ou ausentes, e proporcionar a estimulação elétrica das fibras do nervo auditivo remanescente (BELIVACQUA; COSTA-FILHO; MARTINHO, 2004; COSTA-FILHO, BEVILACQUA, AMANTINI, 2005).

Segundo esses mesmos autores, o benefício mais relevante proporcionado pelo implante coclear é a possibilidade de percepção dos sons de fala de frequências altas. Isto permite que a criança consiga reconhecer auditivamente os sons de fala com mais facilidade, e a aquisição da linguagem oral ocorre mais rapidamente e de maneira menos árdua.

O implante coclear é um dispositivo de amplificação sonora inserido cirurgicamente, que requer avaliação criteriosa na seleção de crianças candidatas e acompanhamento pós-cirúrgico rigoroso, tanto no que se refere aos procedimentos de ativação e mapeamento dos eletrodos, avaliação audiológica e complementares, como também na realização de terapia fonoaudiológica especializada (COSTA-FILHO, BEVILACQUA, AMANTINI, 2005).

Moret, Bevilacqua e Costa-Filho (2007) estudaram o desempenho de audição e de linguagem oral de crianças portadoras de deficiência auditiva sensorioneural profunda bilateral pré-lingual, usuárias de implante coclear multicanal, quanto aos seguintes aspectos: idade da criança na época da realização da pesquisa, tempo de privação sensorial auditiva, tempo de uso do implante coclear, tipo de implante coclear, estratégia de codificação de fala utilizada, grau de permeabilidade da família no processo terapêutico e estilo 
cognitivo da criança. Participaram do estudo 60 crianças que foram avaliadas quanto às categorias de audição e de linguagem. Todas as variáveis foram analisadas estatisticamente. Os aspectos psicossociais, considerando o estilo cognitivo da criança e o grau de permeabilidade da família também foram variáveis investigadas. Quanto ao desempenho de audição e de linguagem com o uso do implante coclear, as categorias auditivas intermediárias e avançadas foram alcançadas por mais da metade do grupo de crianças. Os aspectos estatisticamente significantes no desempenho de audição e de linguagem oral foram: idade da criança na avaliação, o tempo de privação sensorial auditiva, o tempo de uso do implante coclear, o tipo de implante, a estratégia de codificação dos sons da fala e a permeabilidade da família. Com base nesses resultados, concluíram que o implante coclear é altamente efetivo embora complexo pela interação de variáveis que interferem no desempenho da criança implantada, desafiando novos estudos na compreensão da complexidade da implantação em crianças pequenas.

Assim, o fato do individuo fazer uso do implante coclear não assegura o desenvolvimento das habilidades auditivas e de linguagem oral. Todo este complexo processo depende de inúmeros fatores intrínsecos e extrínsecos ao sujeito, tais como capacidade de memória auditiva, adequada estimulação no ambiente familiar, intervenção fonoaudiológica precoce e também uma somatória de todos esses e outros fatores.

Consequentemente, por inicialmente não desenvolverem a linguagem oral, os indivíduos com implante coclear não podem ser privados da possibilidade da aquisição e do desenvolvimento da linguagem, pois podem fazer isso utilizando outro canal - a visão - e outra forma de comunicação - a língua de sinais.

A língua de sinais é considerada uma língua natural para os surdos, já que sua aquisição se dará naturalmente. É uma língua como qualquer outra língua materna, adquirida efetiva e essencialmente no contato com seus falantes.

A ausência de barreiras à sua aprendizagem pelas pessoas surdas se deve ao fato dela possuir modalidade visual-espacial para sua realização: sua produção é realizada através de signos gestuais e espaciais e sua percepção é realizada por meio de processos visuais (FELIPE; MONTEIRO, 2001).

A língua de sinais, uma vez entendida como a língua materna do surdo, deverá ser dentro da escola, o meio de instrução por excelência para o ensino da língua portuguesa escrita (SALLES et al., 2004).

Longe de minimizar o significado da língua de sinais na vida do surdo, é interessante ressaltar que pesquisas sustentam que se uma criança surda puder aprender a língua de sinais da comunidade surda na qual será inserida, 
ela terá mais facilidade em aprender a língua oral-auditiva da comunidade ouvinte (FELIPE; MONTEIRO, 2001).

Desta forma, é desejável que o surdo também adquira a língua oral da comunidade onde vive, o que remete à constatação de que a língua oral seria adquirida como segunda língua. $O$ aspecto mais flagrante na aquisição de uma língua oral como segunda língua pela criança surda é que ela deve adquirir propriedades no nível fonológico e prosódico que seu aparato sensorial auditivo está prejudicado de aprender. No entanto, ela pode ter acesso à representação gráfica dessas propriedades, que é a modalidade escrita da língua oral. $\mathrm{O}$ letramento fornece, portanto, condição para a aquisição da língua oral pelo surdo, o que remete ao processo psicolinguístico da alfabetização e à explicitação e construção das referências culturais da comunidade letrada. Essa tarefa é, porém, menos árdua se a modalidade escrita da língua oral é adquirida como segunda língua, sendo a língua de sinais adquirida como primeira, cabendo desenvolver estratégias de ensino que levem em consideração a situação psicossocial do surdo, em particular sua condição multicultural (SALLES et al., 2004).

O objetivo deste relato é descrever a experiência do atendimento multidisciplinar de uma criança usuária de implante coclear atendida no Serviço de Fonoaudiologia do Hospital das Clinicas da Faculdade de Medicina de Ribeirão Preto pelo Programa de Saúde Auditiva da Divisão de Otorrinolaringologia.

\section{Relato do caso}

B.V.S.R.: oito anos, gênero masculino, residente em Ribeirão Preto SP. Realiza, desde 2006, atendimentos fonoaudiológicos com fonoaudióloga da área de Audiologia Educacional e atendimentos pedagógicos com pedagoga especialista em Educação Especial/Surdez no Serviço de Fonoaudiologia do Hospital das Clinicas da Faculdade de Medicina de Ribeirão Preto. Os atendimentos são realizados duas vezes por semana, com duração de uma hora cada atendimento. B.V.S.R. apresenta perda auditiva profunda em ambos os ouvidos, cuja etiologia provável foi uso de medicação ototóxica para tratamento de uma infecção renal no pós-parto. Além disso, apresentou outros fatores de risco para perda auditiva, como prematuridade ( 27 semanas e 2 dias), baixo peso ao nascer ( $1050 \mathrm{~kg})$ e hiperbilirrubinemia devido à síndrome de Hellp, que acometeu sua mãe durante a gestação.

Começou a usar o aparelho de amplificação sonora individual (AASI) com um ano de idade e realizar terapia fonoaudiologia em outro serviço. Após dois anos de uso do AASI sem obter ganhos auditivos com o aparelho, foi indicado o implante coclear. Realizou cirurgia para o implante coclear aos três anos e quatro meses de idade na orelha direita e iniciou a reabilitação fonoaudiológica segundo abordagem aurioral e acompanhamento pedagógico em nosso serviço. 
Durante um período de dois anos foram mantidos atendimentos fonoaudiológicos e pedagógicos sistematizados, além de constantes orientações aos familiares, no que se refere ao processo de estimulação das habilidades auditivas e da linguagem oral em situações da rotina diária familiar, buscando-se enriquecer os eventos do dia a dia e criar um ambiente adequado de estimulação. A família mostrava-se receptiva e engajada no processo terapêutico.

Mesmo com o uso do implante coclear, a criança apresentava pouca evolução nos aspectos fonoaudiológicos e pedagógicos. Mantinha-se quieto durante as sessões, não se interessava pelas atividades propostas, não compreendia jogos e brincadeiras e demonstrava pouca intenção comunicativa. Embora a função auditiva fosse constantemente estimulada, a criança não avançava nas habilidades, permanecendo na detecção auditiva apenas de sons instrumentais e ambientais e não dos sons de fala. Com um desempenho auditivo inferior comparado ao descrito em literatura para crianças implantadas nas mesmas condições, foi realizado o mapeamento do implante para verificar a impedância (eficiência) dos eletrodos, que mostrou estar em adequado funcionamento, 0 que não correspondia ao comportamento auditivo apresentado.

Devido à criança estar com seis anos de idade, ingressando no ensino fundamental e apresentando baixo desempenho nas habilidades de linguagem $e$ aprendizagem, o caso foi discutido por uma equipe multidisciplinar (psicóloga, médicos, fonoaudiólogas e pedagogas) e decidiu-se modificar a abordagem, optando-se por iniciar ensino de língua brasileira de sinais (LIBRAS).

A criança foi encaminhada para atendimento com monitora e pedagoga surda a fim de que houvesse um input linguístico favorável à aquisição da língua, possibilitado por ambiente de imersão em língua de sinais.

Foi desenvolvido um planejamento em conjunto com a escola (Municipal) na qual a criança passou a frequentar salas de Atendimento Educacional Especializado tendo o apoio de professora de Educação Especial/Surdez e foi disponibilizado um intérprete de LIBRAS / Língua Portuguesa na sala de aula regular.

\section{Resultados}

Após um período de dois anos, contado a partir da aprendizagem de LIBRAS, B.V.S.R. apresentou considerável evolução em todos os aspectos do desenvolvimento.

Atualmente, a criança está frequentando o segundo ano do ensino fundamental em classe regular da rede municipal de ensino.

Quanto aos aspectos pedagógicos, inicialmente a criança não reconhecia letras e números e não se interessava por atividades de leitura e escrita. Com apoio de recursos visuais: datilologia (alfabeto manual) e sinais em LIBRAS, foi 
elaborado um projeto com objetivo de desenvolver as habilidades cognitivas, treino de coordenação motora, identificação e reconhecimento de letras e números. Foram organizadas atividades onde a criança, por meio de ações (mentais e concretas), construía conhecimentos de diferentes naturezas: físicos, lógico-matemáticos e sociais. Quanto ao desempenho no processo de alfabetização, encontra-se na hipótese silábico-alfabética na aprendizagem da Língua Portuguesa em sua modalidade escrita. Em relação às operações matemáticas, apresenta desempenho semelhante aos demais alunos da sua fixa etária e série escolar. Também apresenta adequado conhecimento do conteúdo das demais disciplinas, tais como Ciências, História e Geografia, quando avaliado em LIBRAS.

No que se refere aos aspectos fonoaudiológicos, as habilidades auditivas de linguagem oral e fala continuaram a ser estimuladas, agora com apoio da LIBRAS e de recursos visuais. Nos primeiros atendimentos, o paciente apenas detectava os sons ambientais e ruídos. À medida que os atendimentos foram acontecendo e com a mudança da abordagem, B.V.S.R. passou a detectar e discriminar sons de fala e reconhecer palavras. Para o desenvolvimento da linguagem, foram trabalhados os seguintes aspectos: noção espaço-temporal, sequência dos acontecimentos e aspectos cognitivos. Nos primeiros atendimentos, o paciente não compreendia a noção temporal, explorada por meio do calendário. Atualmente compreende todas as noções temporais. Para os aspectos cognitivos, trabalhou-se raciocínio, memória e conceitos, pois a criança em questão não conseguia jogar e assimilar as regras básicas dos jogos. Através dos atendimentos, compreendeu diversos jogos próprios a sua faixa etária. Adquiriu conceitos de cores, formas, tamanhos e relações espaciais.

Quanto à emissão de fala, notou-se uma mudança de comportamento muito significativa, pois B.V.S.R. passou de uma situação de ausência de oralidade e poucas atitudes comunicativas para situações de uso efetivo da fala.

Atualmente adquiriu a maioria dos fonemas da língua portuguesa, exceto /k/,/g/,/r/ e grupo consonantal/r/ que ainda estão em fase de estimulação. Aumentou o vocabulário e emite espontaneamente palavras de uso cotidiano. Quanto à linguagem, quando avaliado em LIBRAS, apresenta desempenho semelhante ao das crianças da sua faixa etária, no que se refere aos aspectos semânticos e pragmáticos.

\section{Discussão}

A síndrome de Hellp, que acometeu a mãe durante a gestação, é definida como uma forma grave de pré-eclampsia caracterizada por hemólise, elevação das enzimas hepáticas e baixa de plaquetas. A pré-eclampsia é uma doença privativa da gravidez e tem como tríade sintomática característica a hipertensão, o edema e a proteinúria. O prognóstico fetal são neonatos de baixo peso, pré-termos e uma importante mortalidade perinatal sendo de 7 a $60 \%$ (REZENTE; MONTENEGRO, 1995; NEME; PARPINELLI, 2000). 
Assim como descrito, a criança nasceu prematura, com peso inferior a $1500 \mathrm{~g}$, apresentou hiperbilirrubinemia e usou medicação ototóxica. De acordo com os riscos auditivos caracterizados, em 1994 e em 2000 pelo Joint Committee on Infant Hearing, citado por Azevedo (2005), os riscos são: antecedentes familiares de disacusia sensorioneural hereditária, consanguinidade materna, infecções congênitas (rubéola, sífilis, citomegalovirus, herpes e toxoplasmose); malformações craniofaciais, incluindo as de pavilhão auricular e meato acústico externo, peso de nascimento inferior a $1500 \mathrm{~g}$, recém nascido pequeno para idade gestacional, hiperbilirrubinemia - exsanguineotranfusão, uso de medicação ototóxica, meningite bacteriana, apgar de 0 a 4 no $1^{\circ}$ minuto ou 0 a 6 no $5^{\circ}$, ventilação mecânica por mais de 5 dias, síndromes, alcoolismo materno e uso de drogas psicotrópicas na gestação, hemorragia ventricular, permanência em UTI neonatal por mais de 48 horas, convulsões neonatais, otite media recorrente ou persistente por mais de três meses, suspeitam dos familiares de atraso no desenvolvimento de fala, linguagem e audição, traumatismo craniano com perda de consciência ou fratura craniana.

Um dos critérios de seleção de pacientes para implante coclear é o individuo apresentar perda auditiva sensorioneural de grau profundo. Além do tipo e grau da perda, o individuo necessita de diversos outros exames e avaliações fonoaudiológicas, psicológicas, médicas e sociais. Após todos os exames e avaliações, os dados são discutidos em equipe, para a decisão final de o individuo ser ou não candidato ao implante (KOZLOWSKI, 1997).

Uma vez implantado, deverá seguir com atendimento fonoaudiológico especializado, com propósito da aquisição da linguagem oral a partir da percepção auditiva da fala (MORET, 2005).

Como descrito, a criança atendeu a todos os critérios exigidos pré e pós-cirurgia do implante coclear.

Moret, Bevilacqua, Costa-Filho (2007) ressaltaram que poucos estudos investigaram os efeitos do implante coclear sobre domínios múltiplos da criança, como a cognição e o comportamento. Enfatizaram que à medida que 0 implante provê acesso auditivo aos sons da fala e que a criança começa a responder aos mesmos, a habilidade da criança em regular a atenção e o comportamento aumenta consideravelmente, desencadeando uma melhora na interação pais / criança e enriquecendo as experiências comunicativas. Entretanto, consideram que a audição não pode ser vista como único fator no processo de aquisição da linguagem, destacando a qualidade das interações sociais como fator também significativo na constituição da criança como sujeito da linguagem.

Segundo Rabelo (1996) muitas são as crianças que têm apresentado bons resultados através dos métodos orais, mas existem outras que, por contingências próprias como: perda auditiva muito profunda, lento amadurecimento neurológico, problemas emocionais e outros, não desenvolveram comunicação. 
Em vários países, estes mesmos problemas ocorreram, pondo em dúvida a eficácia da abordagem unicamente oral na reabilitação dos surdos.

Trabalhando-se as técnicas orais e gestuais conjugadas é de se esperar que o individuo adquira conceitos de forma mais rápida e efetiva, pois aqueles que não puderem ser adquiridos através da comunicação oral poderão sê-lo pela língua de sinais. Com o desenvolvimento do processo de reabilitação, estes conceitos, adquiridos através dos sinais, serão incorporados ao acervo oral do sujeito. Desta forma, parece que os surdos poderão ser mais bem trabaIhados quanto aos aspectos cognitivos e linguísticos (RABELO, 1996).

Esta mesma autora em um estudo longitudinal de duas crianças surdas, uma atendida com o método oral (menina - dois anos) e a outra com o método misto fundamentado nos princípios da comunicação total (menina dois anos e dez meses), durante doze meses observou que entre as duas crianças não houve diferença quanto à emissão oral. Ambas apresentaram o mesmo nível de dificuldade, tanto para compreenderem quanto para se expressarem verbalmente. A criança trabalhada sob a abordagem oral apresentou um léxico reduzido e seu nível de emissão não ultrapassou holofrases. A comunicação com pais e terapeuta foi artificial e restrita (vocábulos ligados a eventos concretos e eventos presentes). Já a criança sob a abordagem de comunicação total, desenvolveu um léxico bastante ampliado em relação a verbos na recepção e emissão gestual, apresentou frases simples e justapostas e interagiu bem com terapeuta e pais por meio de sinais. Esses dados, apesar de restritos, pois decorrem somente de dois casos, reforçam a posição de que o uso de sinais não prejudica a oralização e favorece a aquisição de conceitos e de comunicação como um todo.

Inúmeros estudos revelam que a criança inicia o seu desenvolvimento cognitivo, que a capacita a pensar sem símbolos verbais, desenvolvendo uma linguagem própria, que forma a base para a percepção de si mesma, e subsequentemente aprende a lidar com as pessoas e com as coisas do seu meio ambiente. No início, suas expressões tomam a forma de gestos e, portanto, desencorajar ou proibir isto é impedir e retardar o desenvolvimento de linguagem receptiva e expressiva. Palavras são símbolos verbais de experiências vividas, que contêm um significado. Deve-se aceitar este sistema de símbolos e gradualmente deixar a criança surda caminhar até o ponto onde ela reconheça que uma linguagem verbal existe (LEIBOVICI, 1996).

\section{Conclusão}

A mudança de abordagem influenciou positivamente na evolução do desenvolvimento da criança, pois a mesma alcançou adequado desempenho em diversas habilidades linguísticas.

O implante coclear vem se tornando, cada vez mais, um recurso para os indivíduos com perda auditiva sensorioneural bilateral de grau profundo, pro- 
porcionando ganhos significativos em relação à percepção auditiva e produção de fala, sendo que em algumas crianças a aquisição da linguagem oral alcança níveis similares a das crianças ouvintes.

Entretanto, nem todos os indivíduos com surdez podem utilizar-se e/ ou beneficiar-se deste recurso.

A aquisição de uma língua oral por surdos remete a questões complexas tanto do ponto de vista cognitivo, quanto do ponto de vista cultural, social e afetivo. È consenso que a língua de sinais constitui-se de uma excelente modalidade para a aquisição da língua pelo surdo. Ao mesmo tempo é desejável que ele adquira a língua oral da comunidade em que vive.

Neste sentido é necessário desenvolver um amplo intercambio de informações e experiências entre profissionais e interessados nessa questão, incluindo-se primordialmente a própria comunidade surda e sua família, a fim de ampliar o conhecimento da realidade do surdo na busca de sua complexa situação linguística e multicultural.

O caso descrito leva-nos a refletir que independente da abordagem utilizada, esta deve oferecer ao individuo meios pelos quais ele possa desenvolver suas habilidades e capacidades. O importante é garantir o uso competente de uma língua que possibilite o estabelecimento de uma identidade e de uma comunicação efetiva com o outro, evitando privações no desenvolvimento da linguagem e da aprendizagem e consequentes déficits psicológicos, sociais, emocionais e educacionais. Cabe aos profissionais que atuam com deficiência auditiva e surdez serem mediadores neste processo, visando ao que é melhor para cada individuo, estando atentos ao seu desenvolvimento, as suas necessidades e potencialidades e tendo sensibilidade e flexibilidade para saber o que deve ser priorizado em cada momento.

\section{Referências}

AZEVEDO, M. F. Avaliação audiológica no primeiro ano de vida. In: LOPES FILHO, O. Tratado de Otorrinolaringologia. Ribeirão Preto: Tecmedd, 2005. cap. 12 , p. 235-258.

BENTO, R. F. et al. Resultados auditivos com o implante coclear multicanal em pacientes submetidos à cirurgia no Hospital das Clínicas da Faculdade de Medicina da Universidade de São Paulo. Revista Brasileira de Otorrinolaringologia. v. 70, n. 5, set./out., p. 632-7,2004. Disponível em: <http://www.scielo.br/ scielo.php?script=sci_arttext\&pid=S0034-7299200400 0500009\&lng=pt\&nrm=is o\&tlng=pt>. Acesso em: 30 nov. 2010.

BEVILACQUA, M. C. COSTA-FILHO, O.A.; MARTINHO, A.C.F. Implante Coclear. In: FERREIRA, L.P.; BEFFI-LOPES, D.M.; LIMONGI, S.C.O. Tratado de Fonoaudiologia. Roca. São Paulo, 2004, cap.60 p.751-760. 
COSTA-FILHO, O. A.; BEVILACQUA, M.C.; AMANTINI, R.C.B. Considerações sobre o implante coclear em crianças. In: BEVILACQUA, M.C.; MORETE, A.L.M. Deficiência auditiva: conversando com familiares e profissionais de saúde. São José dos Campos: Pulso, 2005. cap. 8, p. 123-138.

FELIPE, T. A.; MONTEIRO, M. S. LIBRAS em contexto: curso básico. Brasília: Ministério da Educação e do Desporto/Secretaria de Educação Especial, 2001.

GODINHO, R.; KEOGH, I.; EAVEY, R. Perda auditiva genética. Revista Brasileira de Otorrinolaringologia. v. 69, n. 1, p. 100-104, jan./fev. 2003. Disponível em: <http://www.scielo.br/scielo.php?script=sci_arttext\&pid=S003472992003000100016\&lng=pt\&nrm=iso > . Acesso em: 30 nov. 2010.

JACOB, L. C. B. et al. Monitoramento auditivo na ototoxidade. Revista Brasileira de Otorrinolaringologia. v.72, n.6, p.836-44, nov./dez. 2006. Disponível em: <http://www.scielo.br/scielo.php?script=sci_arttext\&pid=S003472992006000600017\&lng=pt\&nrm=iso\&tlng=pt> . Acesso em:30 nov. 2010.

KOZLOWSKI, L. Seleção de candidatos a um implante coclear. In: Implantes cocleares. Carapicuíba. Pró-Fono, 1997. Cap.5, p.21-23.

KÓS, A. O.; KÓS, M. I. Etiologias das perdas auditivas e suas características audiológicas. In: FROTA. S. Fundamentos em audiologia. Guanabara Koogan. Rio de Janeiro, 2003, cap.10, p.123-140.

LEIBOVICI, Z. Contribuições e Depoimentos In: CICCONE, M. Comunicação total introdução, estratégias, a pessoa surda. 2. ed. Rio de Janeiro. Cultura Médica. 1996, p.78-102.

MORET, A. L. M. Princípios básicos da habilitação da criança deficiente auditiva com implante coclear. In: BEVILACQUA, M.C.; MORETE, A.L.M. Deficiência auditiva: conversando com familiares e profissionais de saúde. São José dos Campos: Pulso, 2005. cap. 13, p. 225-234.

MORET, A. L. M.; BEVILACQUA, M. C.; COSTA-FILHO, O. A. Implante coclear: audição e linguagem em crianças deficientes auditivas pré-linguais. Pró-Fono Revista de Atualização Científica. v. 19, n. 3, p. 295-304, jul/set. 2007,Disponível em: <http://www.revistaprofono.com.br/ojs/index.php/revistaprofono/article/ view/348/223 >. Acesso em: 30 nov. 2010.

NEME, B. PARPINELLI, M. A. Síndromes hipertensivas na gravidez. In: NEME, B. Obstetrícia Básica. São Paulo: Savier, 2000. cap. 33, p. 313-314.

RABELO, A. S. Contribuições e Depoimentos In: CICCONE, M. Comunicação total introdução, estratégias, a pessoa surda. 2. ed. Rio de Janeiro. Cultura Médica. 1996, p. 78-102.

REZENDE, J.; MONTENEGRO, C.A.B. Toxemia gravídica: pré-eclampsia/ eclampsia. In:_. Obstetrícia Fundamental. Rio de Janeiro: Guanabara, 1995. Cap.18, p. 229-241. 
Michelle N. Valadao - Jilma A. Nomura - Debora H. Mazer - Myriam de L. Isaac

SALLES, H. M. M. L. et al. Ensino da língua portuguesa para surdos: caminhos para a prática pedagógica. Brasília: Ministério da Educação. Secretaria de Educação Especial, 2004. 2v.: il. __ (Programa Nacional de Apoio à Educação dos Surdos).

\section{Correspondência}

Michelle Nave Valadao - Rua Dois de Julho n. 816 Vila Tiberio CEP 14050-450, Ribeirão PretoSão Paulo, Brasil.

E-mail:michellenave@yahoo.com-jilmanomura@hotmail.com

Recebido em 12 de janeiro de 2011

Aprovado em 03 de janeiro de 2012 